\title{
How Accurately Can You Suspect Endometrial Carcinoma in PMB?
}

\section{Ilukpitiya IGDC ${ }^{1}$}

\section{${ }^{1}$ Post graduate trainee, Registrar in Gynaecol- ogy and Obstetrics, Professorial Unit, Univer- sity of Sri Jayawardenepura, Colombo South Teaching Hospital, Kalubowila. \\ Corresponding Author - Dr Ilukpitiya IGDC \\ Email-ilukpitiyad@yahoo.com}

\begin{abstract}
Endometrial carcinoma is the second commonest gynaecological carcinoma worldwide, which is more frequently present as postmenopausal bleeding in early stages. Evaluation of endometrial thickness by $2 \mathrm{D}$ and $3 \mathrm{D}$ transvaginal ultrasonography, saline infusion sonography, MRI, blind and hysteroscopic guided endometrial biopsy are the investigations available to evaluate postmenopausal bleeding. Many studies has been conducted to assess and compare the accuracy of each investigation and found to have differences in sensitivity, specificity and predictive values on diagnosing endometrial malignancies. Therefore, systematic analysis of symptoms, risk factors and supportive investigations will give a better prediction of endometrial carcinoma in women presenting with postmenopausal bleeding.
\end{abstract}

\section{Key words}

postmenopausal bleeding, endometrial carcinoma, sensitivity, specificity, predictive value

\section{Introduction}

Endometrial carcinoma is the second most common female genital tract malignancy worldwide, but it is the commonest gynaecological malignancy in western countries as they are having well establish cervical screening programmes for cervical carcinoma, which is the number one gynaecological cancer among other countries ${ }^{(1)}$. In Sri Lanka, endometrial Carcinoma is the eighth commonest carcinoma among females, where cervical and ovarian carcinoma are the leading genital tract malignancies ${ }^{(2,3)}$. Most of the endometrial cancers are diagnosed in early stages, as most of them are presented as post-menopausal bleeding (90\%), even though only $10 \%-15 \%$ of post-menopausal bleeding is associated with endometrial carcinoma ${ }^{(4,5,6,7)}$. According to the data from year 2018 , deaths due to endometrial carcinoma is $1 \%$ of all deaths due to malignancies in Sri Lanka ${ }^{(3)}$.

\section{Pathology}

The most common $(80 \%)$ histological type of endometrial carcinoma is endometrioid adenocarcinoma (type 1), and they are associated with nulliparity, obesity, insulin resistance, and hyper estrogenic environment. They are graded from 1-3 according to the degree of differentiation and nuclear features. Type 2 tumours include serous, squamous, undifferentiated carcinomas, carcinosarcomas and endometrial stromal sarcomas. They are less common, more aggressive and have a poor prognosis. They are associated with old age and not associated with the risk factors for type 1 cancers. Type 1 tumours usually have a background of endometrial hyperplasia and found to have about $50 \%$ of severe atypical endometrial hyperplasia. Endometrial carcinomas are usually primary and very rarely can be secondaries from breast, ovary, lung, gastric, colorectal carcinomas and melanomas. 
Endometrial carcinoma spreads locally into the myometrium, to the cervix and vagina (haematogenous spread as well can be seen in the vagina as drop lesions). Deeper myometrial invasion can penetrate the serosa and involves the parametrial tissues. Endometrial carcinoma spread through the lymphatics to the external iliac, internal iliac, obturator and para-aortic lymph nodes and this spread is directly proportional to the degree of myometrial involvement. Trans-tubal spread is also possible to the peritoneum and to the ovaries. Lungs are the commonest site of haematogenous metastasis and, type 2 endometrial carcinomas have the highest tendency to spread even with a minimum myometrial invasion.

\section{$\underline{\text { Screening and Diagnosis }}$}

Routine mass screening of the population is not practical due to very low prevalence of endometrial carcinoma and its precursor lesions (complex hyperplasia of the endometrium). Therefore, proper assessment of symptomatic females, specially associated with risk factors, is the mainstay of the diagnosis.

The commonest cause for postmenopausal bleeding is atrophic endometritis or vaginitis, accounting for $60-80 \%$ of all cases $^{(30)}$. Exogenous Estrogen contribute in $15-25 \%$, endometrial hyperplasia in $5-10 \%$ and cervical or endometrial polyps in $2-12 \%$ for postmenopausal bleeding. Bleeding may arise from extra genital arears such as urethra, bladder and rectum. The possibility of cervical carcinoma is always should be kept in mind on investigating for postmenopausal bleeding, especially in Sri Lanka, as a third world country. Even the risk is $10-15 \%, 75 \%$ of endometrial carcinomas occur in postmenopausal period and $90 \%$ of them present as postmenopausal bleeding, making them to seek medical advice in early stage of the disease.

Obtaining a comprehensive history including risk factors with proper clinical examination of the lower genital tract, including assessment of the cervix, vagina and perineum in combination with the investigations, will provide a good predictive value than the predictive values of individual component ${ }^{(28,29)}$. The pre-test probability of endometrial carcinoma is reduced from 10-15 $\%$ up to $1 \%$ post-test with negative results. But it varies with the coexisting risk factors and advancing age, where there is $1 \%$ risk if the age is $<50$ years and $25 \%$ if the age is $>80$ years. The risk is $18 \%$ in obese, $21 \%$ in diabetic and $29 \%$ in obese $\&$ diabetic in combination ${ }^{(6)}$

Surgery is the mainstay of management of endometrial carcinoma including, obtaining peritoneal washing for cytology, extra fascial total hysterectomy, bilateral salphingo-oophorectomy, with or without pelvic and para-aortic lymph node dissection which will provide surgico-pathological staging of the disease. Post-operative adjuvant radiotherapy (external beam or brachy-therapy) would be decided depending on the grade, type and the degree of myometrial invasion of the tumour. Still there are some controversies of the management of endometrial carcinoma, where many studies been focused currently on adjuvant chemotherapy, chemoradiation when compared to radiotherapy alone.

The overall 5 year survival of all stages of the endometrial carcinoma is $80 \%$ and it varies with the grade and myometrial invasion. Survival in stage I disease is approximately $85-90 \%$, and it reduces up to $70-75 \%$ in stage II, $45 \%$ in stage III and $<30 \%$ in stage IV, which reflect the importance of early detection of the disease.

\section{Trans Vaginal Sonography (TVS)}

Multiple logistic regression analysis showed that time since menopause and endometrial thickness were statistically significant predictors of endometrial carcinoma ${ }^{(8)}$.

Transvaginal ultrasonography is a known less invasive investigation which is freely available and 
has a very good patient acceptancy. Usually it is sufficient for an initial evaluation of postmenopausal bleeding if the ultrasound images reveal a thin endometrial echo (less than or equal to 4 $\mathrm{mm}$ ). The patient can be reassured, given that an endometrial thickness of $4 \mathrm{~mm}$ or less has a greater than $99 \%$ negative predictive value for endometrial cancer ${ }^{(9)}$. Some studies show $100 \%$ sensitivity when using $4 \mathrm{~mm}$ thickness of the endometrium as the cutoff value, therefore none of the patients found to have endometrial cancer after the ultrasonography became negative ${ }^{(10)}$.

According to systematic analysis done by Gupta JK et al. only 4 studies from 21 , which used $5 \mathrm{~mm}$ as the cutoff endometrial thickness, had high quality criteria. Using the pooled estimates only from these four studies, a positive test result raised the probability of carcinoma from $14 \%$ to $31.3 \%$, while a negative test reduced it to $2.5 \%{ }^{(11)}$. This shows when the thickness of both endometrial layers at $<$ or $=$ to $5 \mathrm{~mm}$, the negative result will rule out endometrial pathology very well, but it should be always corelated with the associated risk factors and recurrence of postmenopausal bleeding. But positive results will not always give an accurate diagnosis of endometrial carcinoma.

The most cited meta-analysis by Smith-Bindman et al. included 5892 women from 35 prospective studies that compared endometrial thickness measured by TVS to presence or absence of endometrial carcinoma on histology ${ }^{(12)}$. At $5 \mathrm{~mm}$ cut-off, the overall summary mean weighted estimates of the sensitivity for detecting endometrial cancer was $96 \%$ for a $39 \%$ false-positive rate. This would reduce a pre-test probability of $10 \%$ for endometrial cancer to a post-test probability of $1 \%$. Therefore, expectant management (without the need for tissue sampling) is recommended for these women.

There is only one study that looked at follow-up of women with PMB and an endometrial thickness of $<$ or $=4 \mathrm{~mm}^{(13)}$. It showed that none of the women with the expectant management developed cancer over 1 year of follow-up. But endometrial malignancy was missed by TVS in 1 patient among 163 patient $(0.6 \%)$ who was diagnosed by cervical cytological examination. If the endometrial thickness is $>4 \mathrm{~mm}$, the presence of fluid in endometrial cavity is a good marker in diagnosing pathological changes in the endometrium. Curcic et al. concluded that, if the endometrial thickness is $<4 \mathrm{~mm}$ no further evaluation is indicated if the clinical picture supports ${ }^{(24)}$.

Sonographic examination of endometrium is not always accurate as there can be inter and intra examiner variation. Level of experience, skill of the examiner and some patient factors are unfavorable for the procedure. Transvaginal ultrasonography is also not always reproducible. Therefore, in all the cases where ultrasonography is not possible, considering the risk factors and continuation of per vaginal bleeding, an alternative method should be advised.

\section{$\underline{\text { Saline Infusion Sonogram (SIS) }}$}

Saline infusion sonogram is done by infusing saline into the uterine cavity using a catheter through the cervix before performing trans vaginal ultrasonogram. This will separate two endometrial lining and allows to measure endometrial thickness more accurately and visualize intracavitary lesions such as polyps and fibroids.

The meta-analysis done by de Kroon et al. on the accuracy of SIS on analyzing the endometrium in patients with abnormal uterine bleeding, concluded that its ability in evaluating uterine cavity in pre and postmenopausal women ${ }^{(17)}$. But it was more feasible on premenopausal women than post menopausal women (success rate $95 \%$ and $86 \%$ respectively). The pooled sensitivity and pooled specificity of SIS in uterine cavity evaluation were $95 \%$ and $88 \%$ respectively, the likelihood ratios were 8.23 and 0.06 respectively and the post-test probabilities were 0.91 and 0.07 respec- 
tively. But this method can be little discomforting among postmenopausal women than conventional TVS reducing patient's acceptance and technical failure can also be encountered.

Gel instillation sonography is a feasible, accurate alternative for SIS in the evaluation of women with abnormal bleeding, and has fewer technical failures $^{(18)}$.

\section{Endometrial Sampling}

Dilatation and curettage was used in the past to obtain endometrial sampling as a blind procedure. But this method is now outdated, as it is considered as a minor surgical procedure which required the patient to get admitted and to undergo general or regional anaesthesia, while there are novel methods that has been developed for endometrial sampling as outpatient procedure. Complications due to anaesthesia, uterine perforation and bleeding are the main possible complication of dilatation and curettage. A Clinical trial conducted by Sanam et al. revealed that dilatation and curettage has $100 \%$ sensitivity, specificity, positive and negative predictive values and accuracy, where the values with Pipelles sampling was comparable with the results which is a very cheap, easy and reliable method with high patient acceptan$\mathrm{cy}^{(25)}$.

The Pipelles device consists of a disposable plastic outer tube measuring $3 \mathrm{~mm}$ in diameter, within it, is a closely fitting rod. When the rod is withdrawn, it creates a vacuum that sucks in a section of endometrium, sufficient to give a histological report. Because of its smaller diameter it is very easier to insert through the cervix without needing any anaesthesia and cervical grasping. A meta-analysis done by Dijkhuizen et al. shows Pipelle device and the Vabra device (an endometrial aspiration device) has shown a very good detection rates on diagnosing endometrial carcinoma $99.6 \%$ and $97.1 \%$ respectively ${ }^{(19)}$. Pipelle was the most sensitive device with the sensitivity of $81 \%$ and the specificity of both methods was $>98 \%$. But insertion of the device may be difficult in postmenopausal women as the cervix gets narrowed and it is not an infrequent thing that the histology report mentions as insufficient sampling.

$6 \%$ out of 66 patients with insufficient sampling were diagnosed to have endometrial carcinoma or atypical hyperplasia subsequently by a prospective study performed by Van Doorn et $\mathrm{al}^{(20)}$. This finding implies that women with an insufficient sample and an endometrial thickness of $5 \mathrm{~mm}$ should not be reassured. The patient can be reassured with inadequate sample if the hysteroscopic and sonographic findings are also reassuring only, according to the controlled regression analysis by Bakour et $\mathrm{al}^{(21)}$.

\section{Hysteroscopy}

Hysteroscopy provides a direct visualization of the endometrial cavity compared to traditional blind procedures. It allows to take selective endometrial sampling only from the suspicious lesions, and therefore having a very low likelihood ratio of endometrial carcinoma when there is a negative hysteroscopy ${ }^{(22)}$. Study conducted by Epstein E et al. on the accuracy of TVS, SIS and Hysteroscopy on diagnosing endometrial pathology revealed that hysteroscopy is always superior to other two methods with regard to differentiating between malignant and benign lesions (sensitivity $84 \%, 44 \%$, and $60 \%$; false-positive rate $15 \%, 6 \%$ and $10 \%$, respectively) ${ }^{(16)}$. Therefore, outpatient hysteroscopy and biopsy are still the methods of choice $^{(17)}$

There are rigid and flexible hysteroscopes that can be used in outpatient procedures. Although flexible hysteroscopy is comparatively less painful, rigid hysteroscopy provides superior optical qualities and higher success rates. A liquid with low viscosity (normal saline) or gas $\left(\mathrm{CO}_{2}\right)$ can be used as expansion medium and continuous flow of fluid will distend the vagina and the cervical canal and allows the easy entry into the endometrial 
cavity. Introduction of very small diameter hysteroscopes $(3 \mathrm{~mm})$, and vaginoscopic non-touch method has reduced insertion of speculum and cervical grasp by tenaculum and has increased patient acceptancy and minimized the requirement of anaesthesia, making the office hysteroscopy as a currently popular method. In some instances, patient can develop pain due to cervical and uterine distension, therefore, a low threshold should be kept for local anaesthesia. Sometimes vision may be poor due to bleeding and fluid collection. In-patient hysteroscopy may be required if the outpatient hysteroscopy is inadequate or difficult. The sensitivity, specificity, accuracy and positive and negative predictive values of hysteroscopy are $94.4 \%, 97.0 \%, 96.8 \%, 68 \%$ and $99.6 \%$, respectively, on diagnosing endometrial carcinoma or hyperplasia according to a systematic quantitative review conducted by Clark et $\mathrm{al}^{(22)}$.

\section{Three-dimensional Ultrasonography}

Three-dimensional ultrasonography will provide a $3 \mathrm{D}$ view of the endometrium and the uterus, needs good technical skills than conventional 2D ultrasonography and availability is also limited. Studies show that the diagnostic performance of $3 \mathrm{D}$ ultrasonography is not superior to $2 \mathrm{D}$ ultrasonogram on differentiating benign and malignant endometrial lesions in women present with postmenopausal bleeding.

\section{MRI (Magnetic Resonance Imaging)}

MRI images help in differentiating endometrial polyps from endometrial malignancies by morphology and accurate detection of myometrial and cervical invasion. It has a mean sensitivity of $79 \%$, specificity of $89 \%$, accuracy of $86 \%$, positive predictive value of $82 \%$, and negative predictive value of $88 \%$ for diagnosis of carcinoma ${ }^{(33)}$. But MRI is not frequently used as a routine investigation in postmenopausal bleeding, as its limited availability and less cost effectiveness compared to other methods.

Table 1. Sensitivity, specificity, positive and negative predictive values of investigations, frequently used for the evaluation of postmenopausal bleeding in diagnosis of endometrial carcinoma.

\begin{tabular}{|l|r|r|r|r|}
\hline Diagnostic test & Sensitivity & Specificity & $\begin{array}{l}\text { positive } \\
\text { predictive } \\
\text { value }\end{array}$ & $\begin{array}{l}\text { Negative } \\
\text { predictive } \\
\text { value }\end{array}$ \\
\hline TVS (ET-4mm as cut off) & $100 \%$ & $61 \%$ & $39 \%$ & $100 \%$ \\
\hline $\begin{array}{l}\text { TVS (ET-5mm as cut } \\
\text { off) }\end{array}$ & $88-95 \%$ & $45-96 \%$ & $31 \%$ & $97.5 \%$ \\
\hline SIS & $95 \%$ & $88 \%$ & $16 \%$ & $97 \%$ \\
\hline Pipelle biopsy ${ }^{(31)}$ & $84-99.6 \%$ & $98-99 \%$ & $94 \%$ & $94 \%$ \\
\hline Novac Curratage & $90-100 \%$ & $100 \%$ & $100 \%$ & $98-100 \%$ \\
\hline $\begin{array}{l}\text { Hysteroscopic guided } \\
\text { biopsy }\end{array}$ & $94 \%$ & $97 \%$ & $68 \%$ & $99.6 \%$ \\
\hline MRI & $79 \%$ & $89 \%$ & $82 \%$ & $88 \%$ \\
\hline
\end{tabular}

(TVS - Trans Vaginal Sonogram, ET - Endometrial Thickness, SIS - Saline Infusion Sonography, MRI - Magnetic Resonance Imaging) 


\section{$\underline{\text { Summary and Conclusion }}$}

As a summary, when a postmenopausal woman comes with bleeding and having negative cervical smear, normal cervix and vagina on speculum examination, Trans-vaginal ultrasonography and endometrial thickness assessment can be offered as first line investigation. Even if the endometrial thickness is less than $4 \mathrm{~mm}$ endometrial sampling can be considered if there are associated risk factors, such as, recurrence of bleeding, fluid in the cavity, irregular endometrium and other risk factors for endometrial carcinoma (lack of evidence yet). If the endometrial thickness is $>4 \mathrm{~mm}$, blind or hysteroscopic guided endometrial sampling, is recommended depending on the availability of the resources and to be managed according to the pathology. If the histopathology sample comes as insufficient, patient can be reassured provided there are hysteroscopic evidence of endometrial atrophy. If there is no evidence of atrophy or recurrence bleeding even with atrophy, inward hysteroscopy and biopsy is recommended for further evaluation ${ }^{(26)}$. The sensitivity, specificity, positive and negative predictive values of each investigation is summarized in Table 1.

\section{$\underline{\text { References }}$}

1. James M, Mohamed K. Endometrial Cancer. Obstetrics, Gynaecology \& Reproductive Medicine 2013; 23:263-269.

2. Samarakoon NK, Siriwikum LBDJ, Liyanage SK, Mahendra BAGG, Rathnayake RMUS, Hewavisenthi SJDeS. The pathological profile of endometrial carcinoma in a cohort of Sri Lankan patients. Journal of Diagnostic Pathology 2016; 11(2)50

3. The global cancer observatory, Sri Lankan data, May 2019, world health organization. Available from: https://gco. iarc.fr/today/data/factsheets/populations/144-sri-lanka-fact-sheets.pdf

4. Astrup K, Olivarius NDF. Frequency of spontaneously occurring postmenopaus- al bleeding in the general population. Acta Obstetricia et Gynaecologica Scandinavica 2004; 83:203-207.

5. Timmermans A, Gerritse MB, Opmeer BC, Jansen FW, Mol BW, Veersema S. Diagnostic accuracy of endometrial thickness to exclude polyps in women with postmenopausal bleeding. Journal of Clinical Ultrasound 2008; 36:286-290.

6. Smith-Bindman R, Kerlikowske K, Feldstein VA, Subak L, Scheidler J, Segal M, et al. Endovaginal ultrasound to exclude endometrial cancer and other endometrial abnormalities. JAMA 1998; 280:1510-1517.

7. Parkin DM, Bray F, Ferlay J, Pisani P. Estimating the world cancer burden: Globocan 2000. International Journal of Cancer 2001; 94:153-156.

8. Van Doorn LC, Dijkhuizen FP, Kruitwagen RF, Heintz AP, Kooi GS, Mol BW. Accuracy of transvaginal ultrasonography in diabetic or obese women with postmenopausal bleeding. Obstetrics and Gynecology 2004; 104:571-578.

9. Scottish Intercollegiate Guidelines Network Investigation of Post- Menopausal Bleeding Section 5: Interpretation of Transvaginal Ultrasound (TVUS). Available from: https:// pdfs.semanticscholar.org/702a/1d879bd13662aba45ddf7bab97d505a4fb16.pdf? ga $=2.218453210 .2112398615 .1597802323$ 1337495100.1597802323

10. Ilan Bruchim MD, Tal Biron-Shental MD, Marco M. Altaras MD, Ami Fishman MD. Combination of endometrial thickness and time since menopause in predicting endometrial cancer in women with postmenopausal bleeding. Journal of Clinical Ultrasound 2004; 32(5)219-224

11. The Role of Transvaginal Ultrasonography in Evaluating the Endometrium of Women 
With Postmenopausal Bleeding - ACOG committee opinion no 734. Available from: https://pubmed.ncbi.nlm.nih.gov/29683909/

12. Peter SMD, Oddvar B, Gun H, Ulf U. Transvaginal Ultrasound for Identifying Endometrial Abnormality. Acta Obstetricia et Gynaecologica Scandinavica 1991; 70(78):591-594. Available from https://doi. org/10.3109/00016349109007922

13. Gupta JK, Chien PF, Voit D, Clark TJ, Khan KS. Ultrasonographic endometrial thickness for diagnosing endometrial pathology in women with postmenopausal bleeding: a meta-analysis Acta Obstetricia et Gynaecologica Scandinavica 2002; 81(9)799-816. Available from https://doi.org/10.1034/ j.1600-0412.2001.810902.x

14. Smith-Bindman R, Kerlikowske K, Feldstein VA, Subak L, Scheidler J, Segal M, et al. Endovaginal ultrasound to exclude endometrial cancer and other endometrial abnormalities. JAMA 1998; 280:1510-1517.

15. Gull B, Carlsson S, Karlsson B, Ylöstalo P, Milsom I, Granberg S. Transvaginal ultrasonography of the endometrium in women with postmenopausal bleeding: is it always necessary to perform an endometrial biopsy? American Journal of Obstetrics and Gynaecology 2000; 182:509-515.

16. American College of Obstetricians and Gynecologists. ACOG Committee Opinion No. 426: The role of transvaginal ultrasonography in the evaluation of postmenopausal bleeding. Obstetrics and Gynaecology 2009; 113(2 Pt 1):462-464.

17. Epstein E, Valentin L. Gray-scale ultrasound morphology in the presence or absence of intrauterine fluid and vascularity as assessed by color Doppler for discrimination between benign and malignant endometrium in women with postmenopausal bleeding. Obstetrics and Gynaecology 2006; 28(1)89-95
18. Epstein E, Ramirez A, Skoog L, Valentin L. Transvaginal sonography, saline contrast sonohysterography and hysteroscopy for the investigation of women with postmenopausal bleeding and endometrium $>5 \mathrm{~mm}$. Ultrasound in Obestetrics and Gynaecology 2001; 18(2):157-162.

19. De Kroon CD, De Bock GH, Dieben SW, Jansen FW. Saline contrast hysterosonography in abnormal uterine bleeding: a systematic review and meta-analysis. BJOG 2003; 110:938-947.

20. Werbrouck E, Veldman J, Luts J, Van HS, Van SD, Timmerman D, Van den Bosch T. Detection of endometrial pathology using saline infusion sonography versus gel instillation sonography: a prospective cohort study. Fertil Steril 2011; 95(1):285-288.

21. Dijkhuizen FP, Mol BWJ, Brölmann HAM, Heintz AP. The accuracy of endometrial sampling in the diagnosis of patients with endometrial carcinoma and hyperplasia: a meta-analysis. Cancer 2000; 89:1765-1772.

22. Van DHC, Opmeer BC, Burger CW, Duk MJ, Kooi GS, Mol BW. Inadequate office endometrial sample requires further evaluation in women with postmenopausal bleeding and abnormal ultrasound results. International Journal of Gynecology and Obstetrics 2007; 99:100-4.

23. Bakour SH, Khan KS, Gupta JK. Controlled analysis of factors associated with insufficient sample on outpatient endometrial biopsy. BJOG 2000; 10:1312-1314.

24. Clark TJ, Voit D, Gupta JK, Hyde C, Song F, Khan KS. Accuracy of hysteroscopy in the diagnosis of endometrial cancer and hyperplasia: a systematic quantitative review. JAMA 2002; 288:1610-1621

25. Camila TR, Júlio CR, Marcos FS, Ana CJ, Omero BP, Francisco JC, Antonio AN. Hys- 
teroscopy as a standard procedure for assessing endometrial lesions among postmenopausal women. Sao Paulo Medical Journal 2007; 125:6. Available from https://doi. org/10.1590/S1516-31802007000600007

26. Curcić A, Durdević S, Mihaldzić-Tubić S, Mladenović-Segedi L, Maksimović M. Ultrasound detection of endometrial fluid in postmenopausal women. Medicinski Pregled 2009; 62:337-341.

27. Sanam M, Majid MMK, Comparison the Diagnostic Value of Dilatation and Curettage Versus Endometrial Biopsy by Pipelle - a Clinical Trial, Asian PacificJournal of Cancer Prevention 2015; 16(12)49714975. Available from DOI:10.7314/apjcp.2015.16.12.4971

28. Shagaf HB, Anne T, Ben WM, Khalid SK. Management of women with postmenopausal bleeding: evidence-based review. The Obstetrician and Gynaecologist 2012 ; 14(4)243-249. Available from DOI: $\quad 10.1111 /$ j.1744-4667.2012.00129.x 2012;14:243-249

29. Ziegler D. Contrast ultrasound: a simple-touse phase-shifting medium offers saline infusion sonography-like images. Fertil Steril. 2009; 92(1):369-373. Available from doi: 10.1016/j.fertnstert.2008.04.032.

30. Khan KS, Dinnes J, Kleijnen J. Systematic reviews to evaluate diagnostic tests. European Journal of Obstetrics and Gynecology and Reproductive Biology 2001; 95:6-11.

31. Khan KS, Chien PFW, Dwarakanath LS. Logistic regression models in obstetrics and gynaecology literature. Obstetrics and Gynecology 1999; 93:1014-1020.

32. Mohamed O, Joanna F, Jackie R, Hizbullah $\mathrm{S}$, Jemma J. Endometrial pathology in the postmenopausal woman - an evidence based approach to management. The Obstetrician
\& Gynaecologist 2015; 17:29-38

33. Machado F, Moreno J, Carazo M, León J, Fiol G, Serna R, Accuracy of endometrial biopsy with the Cornier pipelle for diagnosis of endometrial cancer and atypical hyperplasia. European Journal of Gynaecological Oncology 2003; 24(3-4):279-281

34. Bruno C, Tiina R, Pentti L, Pekka Y. Transvaginal sonography and hysteroscopy in postmenopausal bleeding. Acta Obstetricia et Gynaecologica Scandinavica 1994; 73(5) 413-416. Available from https://doi. org/10.3109/00016349409006254

35. Ralf PG, Eric KO, Evan SS, David C, Laurence $\mathrm{P}$, Shahid $\mathrm{MH}$. Endometrial Polyps: MR Imaging Features and Distinction from Endometrial Carcinoma. Radiology 2000; 214(1): Available from https://doi. org/10.1148/radiology.214.1.r00ja3647 